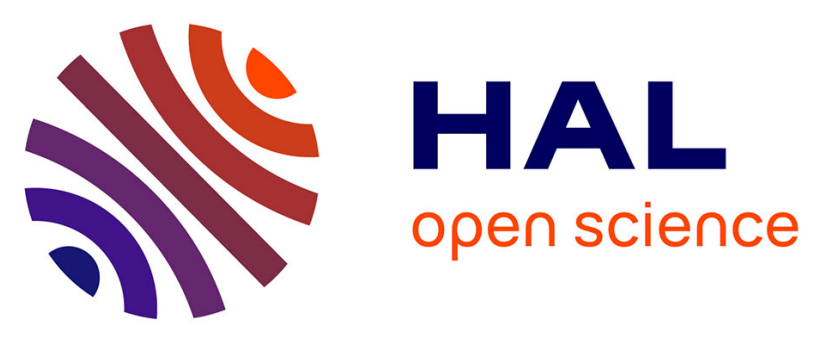

\title{
An Interactive Web-Tool for Molecular Analyses links Naturally Occurring Mutation Data with Three-Dimensional Structures of the rhodopsin-like Glycoprotein Hormone Receptors
}

Gunnar Kleinau, Annika Kreuchwig, Catherine Worth, Gerd Krause

\section{To cite this version:}

Gunnar Kleinau, Annika Kreuchwig, Catherine Worth, Gerd Krause. An Interactive Web-Tool for Molecular Analyses links Naturally Occurring Mutation Data with Three-Dimensional Structures of the rhodopsin-like Glycoprotein Hormone Receptors. Human Mutation, 2010, 31 (6), 10.1002/humu.21265 . hal-00552387

\section{HAL Id: hal-00552387 \\ https://hal.science/hal-00552387}

Submitted on 6 Jan 2011

HAL is a multi-disciplinary open access archive for the deposit and dissemination of scientific research documents, whether they are published or not. The documents may come from teaching and research institutions in France or abroad, or from public or private research centers.
L'archive ouverte pluridisciplinaire HAL, est destinée au dépôt et à la diffusion de documents scientifiques de niveau recherche, publiés ou non, émanant des établissements d'enseignement et de recherche français ou étrangers, des laboratoires publics ou privés. 


\section{Human Mutation}

WILEY

\section{An Interactive Web-Tool for Molecular Analyses links Naturally Occurring Mutation Data with Three-Dimensional Structures of the rhodopsin-like Glycoprotein Hormone Receptors}

\begin{tabular}{|r|l|}
\hline Journal: & Human Mutation \\
\hline Manuscript ID: & humu-2010-0057.R1 \\
\hline Datey - Manuscript type: & Database in Brief \\
\hline Author: & 31 -Mar-2010 \\
\hline Key Worde List of Authors: & $\begin{array}{l}\text { Kleinau, Gunnar; Leibniz-Institut für Molekulare Pharmakologie } \\
\text { (FMP); Charité Universitätsmedizin, Institute of Experimental } \\
\text { Pediatric Endocrinology } \\
\text { Kreuchwig, Annika; Leibniz-Institut für Molekulare Pharmakologie } \\
\text { (FMP) } \\
\text { Worth, Catherine; Leibniz-Institut für Molekulare Pharmakologie } \\
\text { (FMP) } \\
\text { Krause, Gerd; Leibniz-Institut für Molekulare Pharmakologie (FMP) }\end{array}$ \\
\hline Naturally occurring mutations, Database, Glycoprotein hormone \\
\hline
\end{tabular}

\section{ScholarONE \\ Manuscript Central}




\title{
An Interactive Web-Tool for Molecular Analyses Links Naturally Occurring Mutation Data with Three- Dimensional Structures of the Rhodopsin-like Glycoprotein Hormone Receptors
}

\author{
Gunnar Kleinau ${ }^{1,2,+}$, Annika Kreuchwig ${ }^{1,+}$, Catherine L. Worth ${ }^{1}$, and Gerd Krause ${ }^{1, *}$ \\ ${ }^{1}$ Leibniz-Institut für Molekulare Pharmakologie (FMP), Berlin, Germany; ${ }^{2}$ Institute of Experimental Pediatric Endocrinology, \\ Charité Universitätsmedizin Berlin, Germany \\ +denotes equal contribution
}

${ }^{*}$ Correspondence to Gerd Krause, Leibniz-Institut für Molekulare Pharmakologie, Robert-Rössle-Str.10, D-13125 Berlin, Germany, Tel: xx-49-30-94793 228, Fax: xx-49-30-94793 169, E-mail: gkrause@ fmp-berlin.de

Contract grant sponsor: Deutsche Forschungsgemeinschaft; Contract grant number: KR1273/4-1; KL2334/2-1

Communicated by Bruce Gottlieb

\begin{abstract}
The collection, description and molecular analysis of naturally occurring (pathogenic) mutations are important for understanding the functional mechanisms and malfunctions of biological units such as proteins. Numerous databases collate a huge amount of functional data or descriptions of mutations, but tools to analyse the molecular effects of genetic variations are as yet poorly provided. The goal of this work was therefore to develop a translational web-application that facilitates the interactive linkage of functional and structural data and which helps improve our understanding of the molecular basis of naturally occurring gain- or loss- of function mutations. Here we focus on the human glycoprotein hormone receptors (GPHRs), for which a huge number of mutations are known to cause diseases. We describe new options for interactive data analyses within three-dimensional structures, which enable the assignment of molecular relationships between structure and function. Strikingly, as the functional data are converted into relational percentage values, the system allows the comparison and classification of data from different GPHR subtypes and different experimental approaches. Our new application has been incorporated into a freely available database and website for the GPHRs (http://www.ssfa-gphr.de), but the principle development would also be applicable to other macromolecules. O2010 Wiley-Liss, Inc.
\end{abstract}

KEY WORDS: Naturally occurring mutations, Database, Glycoprotein hormone receptors, TSHR, FSHR, LHCGR

\section{INTRODUCTION}

Analyses of naturally occurring mutations are important for understanding the molecular mechanisms and malfunctions of biological macromolecules such as the G-protein-coupled receptors (GPCRs). These receptors are

Received 3 February 2010; accepted revised manuscript 2 April 2010.

(C) 2010 WILEY-LISS, INC. 


\section{Kleinau et al.}

the largest family of membrane proteins and mediate most cellular responses to hormones and neurotransmitters, as well as being responsible for vision, olfaction and taste (reviewed in (Schwartz et al., 2006)). Malfunction of GPCRs causes a variety of human diseases (reviewed in (Schoneberg et al., 2002; Tao, 2006)) and it has been estimated that GPCRs represent between 25-50\% of current drug targets (Overington et al., 2006; Kristiansen, 2004; Flower, 1999). Investigation of mutations provides a powerful tool for deciphering causal relationships between such biological structures and patient phenotypes (Schoneberg et al., 2002; Schoneberg et al., 2004) and is also a prerequisite for the directed development of pharmacological treatments.

Several databases collate information and functional data on GPCRs: GPCR Database, http://www.gpcr.org/7tm (Horn et al., 1998); GRIS: Glycoprotein hormone Receptor Information System, http://gris.ulb.as.be (Van Durme et al., 2006); TSH Receptor mutation Database II, http://innere.uniklinikum-leipzig.de/tsh (Lachmund et al., 2004; Fuhrer et al., 2003); GPCR Natural Variants Database, http://nava.liacs.nl (Kazius et al., 2008). The unification and assignment of such information directly to the three-dimensional (3D) structure of GPCRs would facilitate the elucidation of causal relationships between sequence, structure, function and also malfunction. A direct and interactive link between mutation data and 3D structures would allow the molecular reasons for malfunction (which are ultimately responsible for patient phenotypes) to be studied more precisely.

Therefore we developed a web-application that facilitates interactive linkage between unified functional and 3D structural information. Our project focused on pathogenic mutations of the glycoprotein hormone receptors (GPHRs), which are available in a freely accessible database (http://www.ssfa-gphr.de), where our new tool is incorporated. A large number of GPHR mutations are known to cause various diseases such as hyperthyroidism (thyrotropin receptor, TSHR), hypogonadism (follitropin receptor, FSHR) or precocious puberty (lutropin/choriogonadotropin receptor, LHCGR). The system allows the comparison and semi-quantitative classification of mutant data from different GPHR subtypes and different experimental approaches. In our database and web-application the available structures (fragments) and homology models of GPHRs are used for projection of functional information on to 3D structures in order to facilitate molecular and interactive sequence-structurefunction analyses of pathogenic mutations and receptor malfunction.

\section{THE DATABASE AND NEW ANALYSIS TOOLS}

\section{Background}

Published data and information on the thyrotropin receptor (TSHR), follitropin (FSHR) and lutropin (LHCGR) receptors are collected in a web accessible database (www.ssfa-gphr.de). We updated and redesigned our database (SSFA-GPHR) and web-interface, which combines protein sequences, multiple sequence alignments, protein structures, homology models and data from mutagenesis experiments of GPHRs (Kleinau et al., 2007a). As the functional data are converted into relational percentage values, the system allows the comparison and semiquantitative classification of mutagenesis data from different GPHR subtypes and different experimental approaches. We implemented new data, types of data (e.g. double mutations) and bioinformatic tools like 3D search tools.

\section{Interactive linkage of functional information with three-dimensional structures}

One section of the database presents germline and somatic single side-chain substitutions of the GPHRs (website-link: "Natural mutations"). In a sub-section ("3D structural-search") we provide a novel 3D search tool whereby positions of known naturally occurring mutations are automatically highlighted on homology models of the leucine-rich repeat domain (LRRD), the serpentine domain (SD) (modeling procedures have been previously described in detail (Kleinau et al., 2007a; Kleinau et al., 2007b; Kleinau et al., 2008) or known crystal structures of receptor parts (Fan and Hendrickson, 2005; Sanders et al., 2007). These positions are colored according to the occurrence of inactivating or activating mutations (Figure 1A). Such residues are linked with specific information such as the type of mutation, converted functional data into relational percentage values, or the original source of publication. This information can be retrieved by clicking on the interactive links at the colored C-alpha atoms of mutated positions (Figure 1B, yellow sphere), which leads to a results-overview in a separate browser-window (Figure 1C). In this website section we also added a tabulated overview and a sequence alignment where positions of pathogenic mutations are colored according to their mutation phenotype (gain- or loss- of function). 


\section{Technical data}

The Sequence-Structure-Function-Analysis of Glycoprotein hormone receptors application was built by combining an Apache web server (http://www.apache.org), PHP Hypertext Pre-processor scripts (PHP 5) and a relational database management system (MySQL 5). The web interface is based on HTML, JavaScript and CSS (Cascading Style Sheets). 3D structures are displayed using the Jmol structure viewer (Jmol: an open-source Java viewer for chemical structures in 3D. http://www.jmol.org/). To display the GPHRs in 3D, the viewer is embedded in the application as a Java applet. The Jmol scripting language, in combination with JavaScript, is used to change the rendering of the molecules in order to illustrate important structural features and to allow the linkage of the crystal structures or homology models to the functional information stored in the database.

\section{LINKAGE BETWEEN STRUCTURAL AND FUNCTIONAL INFORMATION OF GLYCOPROTEIN HORMONE RECEPTORS}

The three glycoprotein hormone subtypes are of high physiological importance. Whereas the thyroidstimulating hormone (TSH) regulates growth and function of thyroid follicular cells, the gonadotrophic hormones LH/CG and follicle-stimulating hormone (FSH) play an important role in human reproduction (Ascoli et al., 2002; Rapoport et al., 1998; Simoni et al., 1997; Szkudlinski et al., 2002). Therefore their receptors, TSHR, FSHR, and LHCGR (review (Kleinau and Krause, 2009)), are of central importance to medical, pharmaceutical and biological research (reviewed in (Corvilain et al., 2001; Rapoport and McLachlan, 2007; Schoneberg et al., 2004; Simoni et al., 1997; Smit et al., 2007; Tao, 2006; Tao, 2008; Davies et al., 2005).

We designed an information resource to collect data for the GPHRs and made them available for investigation of sequence-structure-function relationships (Kleinau et al., 2007a). It provides a condensed overview of functional data from mutagenesis studies across the GPHRs using converted relational percentage values and allows searching of the database via different specific tools. This information resource and publicly accessible web interface (http://www.ssfa-gphr.de) was updated and redesigned to contribute to improve the analyses and understanding of malfunctions caused by mutations. We designed and provide web-access to known 3D structures or structural homology models of the receptors with the positions of pathogenic mutations highlighted in different colors according their different function (e.g. gain or loss of function) and also provide links to information from published reports. Furthermore, we have introduced a novel interactive search tool. User-driven picking of particular amino acid positions directly on a 3D structure or homology model provides the user with information about side-chain variations and their particular functional effects. Different effects can be classified and highlighted in different colors. This allows interactions of residues located close in space to be investigated and the comparison of functional data of mutations. Thus, conclusions concerning potential interactions can be drawn according to comparable mutant receptor phenotypes or attractive/repulsive side-chain effects.

Examples of different scenarios for exploring gain-of-function mutations are given in Figure 2A. The first example comprises experimentally validated interacting wild type amino acids (Karges et al., 2005) at which positions mutations are known that lead to a gain-of-function phenotype (wild type residues Val509 in TMH3 and Ala593 in TMH5). The two wild-type amino acids can be seen to interact in the 3D TSHR model. The second example focuses on three tightly-packed triad-like interacting hydrophobic residues, which constitute an hydrophobic patch for stabilization of the basal TSHR conformation. Several naturally occurring single CAMs are known at these positions (Figure 2B) and might be related to hyperfunctioning thyroid nodules (Alberti et al., 2001; Esapa et al., 1999; Gozu et al., 2006; Holzapfel et al., 1997; Kosugi et al., 2000; Trulzsch et al., 2001).

Each of these CAMs causes disruption of the interacting triad, leading to constitutive TSHR activation. This important core of amino acid interaction and molecular interrelationship has not yet been experimentally investigated in detail but according to our model is suggested to be an essential component of the observed mutant phenotypes. Further information regarding the particular amino acids and mutation of interest can be obtained by following the interactive links implemented in our tool. 


\section{Kleinau et al.}

A)
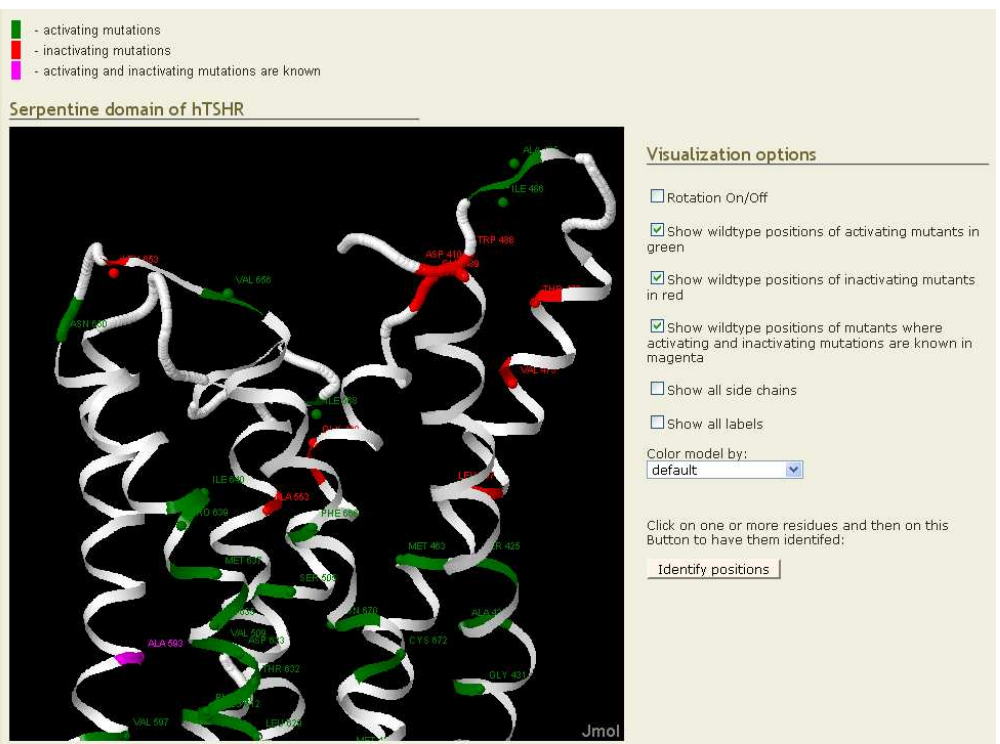

Vhow wildtype positions of activating mutants in green Q Show wildtype positions of inactivating mut
in red WShow wildtype positions of mutants where
activating and inactivating mutations are known magenta

$\square$ show all labels Color model by:
default Click on one or more residues and then on this Identify positions

B)

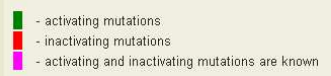

Serpentine domain of hTSHR

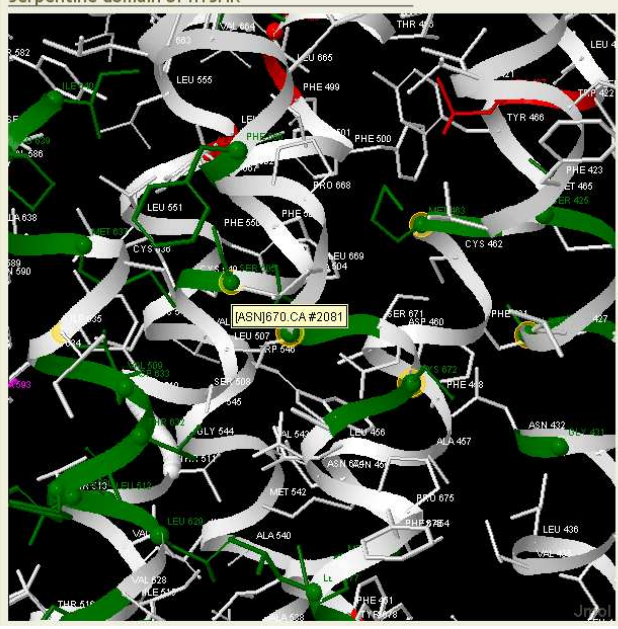

Visualization options

$\square$ Rotation On/off

చshow wildtype positions of actiyating mutants in green Vshow wildtype positions of inactivating mutants ■show wildtype positions of mutants where Show willtype positions of mutants where
activating and inactivating mutations are known in
magenta ఐshow all side chains \Show all labels Color model by:

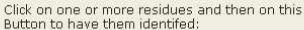
Identify positions Positions you just selected ater Positions you just select
1. alanine -428
2. methionine -463 2. methionine -463
3. serine -505
4. isoleucine -635
5. asparanine -67 4. Isoleucine -635
5. asparagine -670
6. cysteine -672

C)

\section{- Results for 3D - Structural Search}

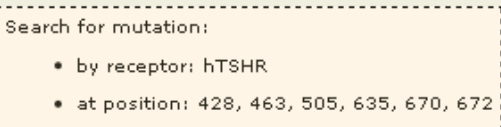

Results

hTSHR Swissprot $3 \mathrm{~d}$ model

Substructure No Ba-We wildtype mutant pubmed

TMH1 4281.46 Ala Val no data available Borgel K. Pohlenz ]...

TMH2 4632.53 Met Val details

TMH3 5053.36 Ser Asn details Holzapfel HP, Wonero..

TMH3 5053.36 Ser Arg no data available Van Sande J, Parma J...

TMH6 6356.46 Ile Val details Castro I, Lima L, Se...

TMH7 6707.45 Asn Ser details Tonacchera M, Van Sa...

TMH7 6727.47 Cys Thr no data available Duprez L, Parma J, V...

Figure 1. Sections of the SSFAGPHR database for investigation, visualization and data collection of naturally occurring single sidechain variations of GPHRs. Three types of search tools are provided: 1. From an alignment of human TSHR, FSHR and LHCGR; 2. from a tabulated and referenced overview and 3. by selecting a structural domain (the leucine-rich repeat domain or serpentine domain). A) Highlighted and colordifferentiated mutations of hTSHR are displayed on a 3D homology model. Wild type positions of reported naturally occurring mutations ("gain-" (green) and "loss- of function" (red) mutations) are shown. Several additional visualization options allow the user to customize how the molecule is displayed, for example color coding of different types of mutations and display/un-display switches for side-chains are provided. B) User-driven picking of selected side chains (yellow sphere) on the 3D structure will retrieve mutation information of each residue. C) Such a request will lead to a result overview opening in a separate browser window and presenting all available information. 
2

3

4

5

6
A)

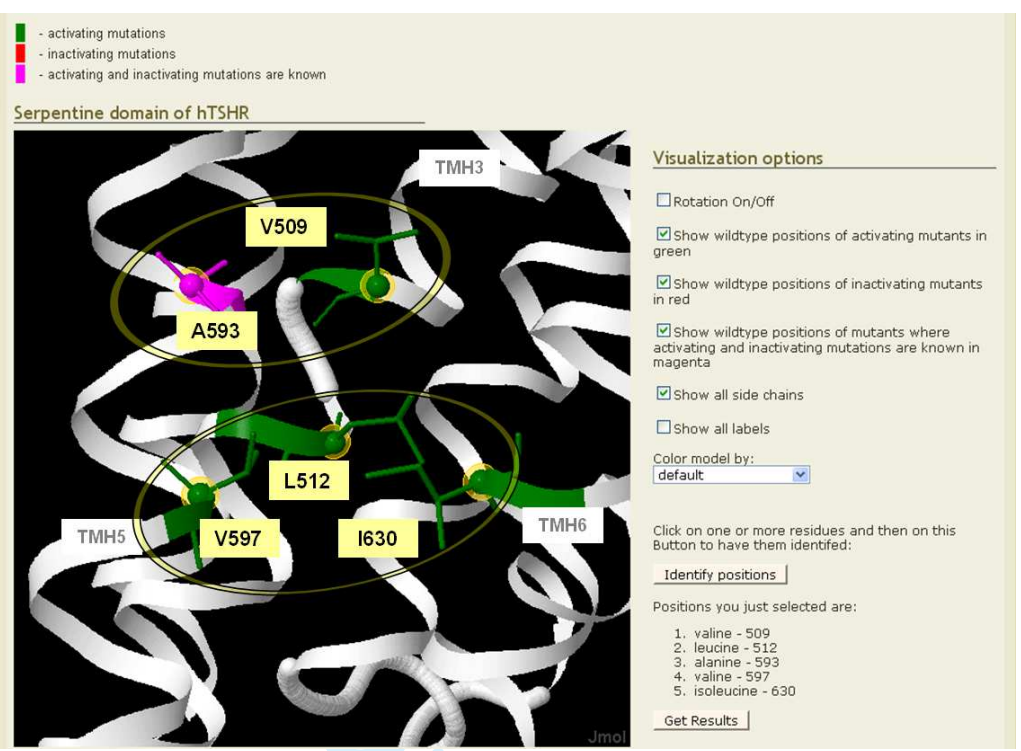

B)

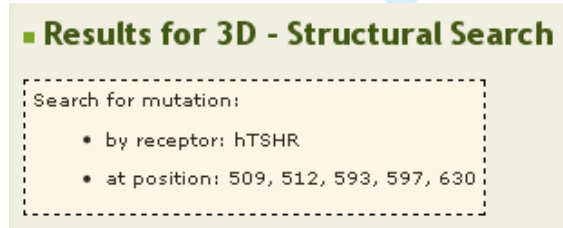

Results

\section{hTSHR Swissprot $3 \mathrm{~d}$ model}

Substructure No Ba-We wildtype mutant

TMH3 $5093.40 \mathrm{Val}$ Ala detail

TMH3 $5093.40 \mathrm{Val}$ Ala details

TMH3 5123.43 Leu Gln details

TMH3 5123.43 Leu Arg details

TMH5 5935.50 Ala Asn details
TMH5 5935.50 Ala

TMH5 5975.54 Val

TMH5 5975.54 Val

TMH6 $6306.41 \quad$ Ile

TMH6

Val

Phe

Leu

Leu

no data

details

details

6306.41 Ile Met details

C)

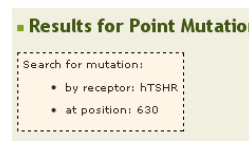

Highlight result values

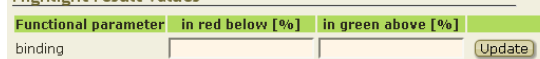

Functional parameter in red below [\%] in green above $[\%]$
binding

Gs_basal activation 179 Update

Gs_max stimulation $\longdiv { 7 9 } \overline { 8 0 }$

expression

Gq_basal activation $\longdiv { 1 7 9 } \longdiv { 1 8 0 }$ Update

\begin{tabular}{l|l|l|l|}
\hline Gq_max stimulation & 79 & 180 & \\
\hline
\end{tabular}

Results

hTSHR Swissprot 3d model 3d morph.

Substructure No Ba-We wildtype mutant binding $\begin{gathered}\text { Gs basal } \\ \text { activation }\end{gathered}$

$\begin{array}{llllll}\text { TMH6 } & 6306.41 & \text { Ile Leu details } 62 & 250 \\ \text { TMH6 } & 6306.41 & \text { Lle }\end{array}$ TMH6 6306.41 Ile Leu details $42 \quad 476$ \begin{tabular}{lllllll}
\hline TMH6 & 6306.41 & Ile & Leu & details - & 640 \\
TMH6 & 6306.41 & Ile & Met & details 46 & 500
\end{tabular}

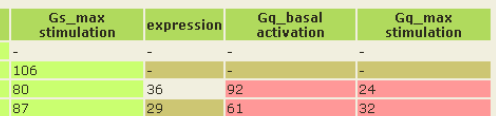

Figure 2. Interacting wild type amino acids of known naturally occurring constitutively activating mutations mapped on to a 3D TSHR model. Five wild type amino acids with known naturally occurring activating mutations are highlighted after performing a 3D search (interactive mouse-click, yellow sphere) A) Double mutation experiments have shown that two of the residues interact with one another (Karges et al., 2005): Val509 in TMH3 and Ala593 in TMH5 (magenta). In spatial neighbourhood a triad of interacting hydrophobic wild type residues Leu512 (TMH3), Ile630 (TMH6) and Val597 (TMH5) can be observed on this $3 \mathrm{D}$ projection, but has not yet been investigated experimentally in detail. It can be speculated that this patch stabilizes the basal conformation of TSHR and that interruption of this interaction by mutation (thereby resulting in a release of flexibility between the helices) leads to TSHR activation. B) By pressing the "Identify positions" and "Get result" buttons detailed information regarding the single substitutions e.g. known mutations identified in vivo, are provided. The source of information from the original publication can be reached via Pubmed links. C) Additionally, mutagenesis data obtained by in vitro studies for the specific amino acid of interest can be retrieved via the 'details' link, if available. Here, a direct comparison of the mutation data (converted into relational percentage values, wt $=$ $100 \%$ is possible using the highlighting feature. The user defined red/green classes indicate that the pathogenic mutations cause strong "gain of function" by constitutively activating effects on the cAMP pathway (> $180 \%$ : increased basal Gs; $>80 \%$ Gs max. stimulation, green) but in fact also a "loss of function" on the IP pathway (<180\%: (not) increased basal Gq; $<80 \%$ Gq max. stimulation, red). 


\section{Kleinau et al.}

Our new tool is therefore suitable for exploring and recognizing spatial interrelationships between side-chains and causally-induced malfunctions of proteins. The 3D mapping and visualization can be used to: $\boldsymbol{a}$. evaluate mutation as already known or as new, $\boldsymbol{b}$. get entrance to published information, $\boldsymbol{c}$. identify direct interaction partners, $\boldsymbol{d}$. evaluate potential mechanisms of molecular malfunction caused by specific mutations. Strikingly, under respect of converted data into comparable percentage values the discrimination of loss- and gain- of function mutations mapped onto the 3D structures helps to both identify hot-spots of signaling mechanisms and integrate and classify new results. Notably, this feature is of universal principle and is not restricted to the GPHRs or other GPCRs, it is also applicable to molecules like DNA or RNA. This tool may help link different scientific fields like biology, biochemistry, medicine, chemistry, genetics and bioinformatics.

\section{REFERENCES}

Alberti L, Proverbio MC, Costagliola S, Weber G, Beck-Peccoz P, Chiumello G, Persani L. 2001. A novel germline mutation in the TSH receptor gene causes non-autoimmune autosomal dominant hyperthyroidism. Eur J Endocrinol 145:249-254.

Ascoli M, Fanelli F, Segaloff DL. 2002. The lutropin/choriogonadotropin receptor, a 2002 perspective. Endocr Rev 23:141174.

Corvilain B, Van Sande J, Dumont JE, Vassart G. 2001. Somatic and germline mutations of the TSH receptor and thyroid diseases. Clin Endocrinol (Oxf) 55:143-158.

Davies TF, Ando T, Lin RY, Tomer Y, Latif R. 2005. Thyrotropin receptor-associated diseases: from adenomata to Graves disease. J Clin Invest 115:1972-1983.

Esapa CT, Duprez L, Ludgate M, Mustafa MS, Kendall-Taylor P, Vassart G, Harris PE. 1999. A novel thyrotropin receptor mutation in an infant with severe thyrotoxicosis. Thyroid 9:1005-1010.

Fan QR, Hendrickson WA. 2005. Structure of human follicle-stimulating hormone in complex with its receptor. Nature 433:269-277.

Flower DR. 1999. Modelling G-protein-coupled receptors for drug design. Biochim Biophys Acta 1422:207-234.

Fuhrer D, Lachmund P, Nebel IT, Paschke R. 2003. The thyrotropin receptor mutation database: update 2003. Thyroid 13:1123-1126.

Gozu HI, Bircan R, Krohn K, Muller S, Vural S, Gezen C, Sargin H, Yavuzer D, Sargin M, Cirakoglu B, Paschke R. 2006. Similar prevalence of somatic TSH receptor and Gsalpha mutations in toxic thyroid nodules in geographical regions with different iodine supply in Turkey. Eur J Endocrinol 155:535-545.

Holzapfel HP, Fuhrer D, Wonerow P, Weinland G, Scherbaum WA, Paschke R. 1997. Identification of constitutively activating somatic thyrotropin receptor mutations in a subset of toxic multinodular goiters. J Clin Endocrinol Metab 82:4229-4233.

Horn F, Weare J, Beukers MW, Horsch S, Bairoch A, Chen W, Edvardsen O, Campagne F, Vriend G. 1998. GPCRDB: an information system for $\mathrm{G}$ protein-coupled receptors. Nucleic Acids Res 26:275-279.

Karges B, Krause G, Homoki J, Debatin KM, de Roux N, Karges W. 2005. TSH receptor mutation V509A causes familial hyperthyroidism by release of interhelical constraints between transmembrane helices TMH3 and TMH5. J Endocrinol 186:377-385.

Kazius J, Wurdinger K, van Iterson M, Kok J, Back T, Ijzerman AP. 2008. GPCR NaVa database: natural variants in human G protein-coupled receptors. Hum Mutat 29:39-44.

Kleinau G, Brehm M, Wiedemann U, Labudde D, Leser U, Krause G. 2007a. Implications for molecular mechanisms of glycoprotein hormone receptors using a new sequence-structure-function analysis resource. Mol Endocrinol 21:574-580.

Kleinau G, Claus M, Jaeschke H, Mueller S, Neumann S, Paschke R, Krause G. 2007b. Contacts between extracellular loop two and transmembrane helix six determine basal activity of the thyroid-stimulating hormone receptor. J Biol Chem 282:518-525. 
Kleinau G, Jaeschke H, Mueller S, Worth CL, Paschke R, Krause G. 2008. Molecular and structural effects of inverse agonistic mutations on signaling of the thyrotropin receptor--a basally active GPCR. Cell Mol Life Sci 65:3664-3676.

Kleinau G, Krause G. 2009. Thyrotropin and homologous glycoprotein hormone receptors: structural and functional aspects of extracellular signaling mechanisms. Endocr Rev 30:133-151.

Kosugi S, Hai N, Okamoto H, Sugawa H, Mori T. 2000. A novel activating mutation in the thyrotropin receptor gene in an autonomously functioning thyroid nodule developed by a Japanese patient. Eur J Endocrinol 143:471-477.

Kristiansen K. 2004. Molecular mechanisms of ligand binding, signaling, and regulation within the superfamily of G-proteincoupled receptors: molecular modeling and mutagenesis approaches to receptor structure and function. Pharmacol Ther 103:21-80.

Lachmund P, Nebel IT, Fuhrer D, Paschke R. 2004. The pedigree tool: web-based visualization of a family tree. Hum Mutat 23:103-105.

Overington JP, Al Lazikani B, Hopkins AL. 2006. How many drug targets are there? Nat Rev Drug Discov 5:993-996.

Rapoport B, Chazenbalk GD, Jaume JC, McLachlan SM. 1998. The thyrotropin (TSH) receptor: interaction with TSH and autoantibodies. Endocr Rev 19:673-716.

Rapoport B, McLachlan SM. 2007. The thyrotropin receptor in Graves' disease. Thyroid 17:911-922.

Sanders J, Chirgadze DY, Sanders P, Baker S, Sullivan A, Bhardwaja A, Bolton J, Reeve M, Nakatake N, Evans M, Richards T, Powell M, Miguel RN, Blundell TL, Furmaniak J, Smith BR. 2007. Crystal structure of the TSH receptor in complex with a thyroid-stimulating autoantibody. Thyroid 17:395-410.

Schoneberg T, Schulz A, Biebermann H, Hermsdorf T, Rompler H, Sangkuhl K. 2004. Mutant G-protein-coupled receptors as a cause of human diseases. Pharmacol Ther 104:173-206.

Schoneberg T, Schulz A, Gudermann T. 2002. The structural basis of G-protein-coupled receptor function and dysfunction in human diseases. Rev Physiol Biochem Pharmacol 144:143-227.

Schwartz TW, Frimurer TM, Holst B, Rosenkilde MM, Elling CE. 2006. Molecular mechanism of 7TM receptor activation--a global toggle switch model. Annu Rev Pharmacol Toxicol 46:481-519.

Simoni M, Gromoll J, Nieschlag E. 1997. The follicle-stimulating hormone receptor: biochemistry, molecular biology, physiology, and pathophysiology. Endocr Rev 18:739-773.

Smit MJ, Vischer HF, Bakker RA, Jongejan A, Timmerman H, Pardo L, Leurs R. 2007. Pharmacogenomic and structural analysis of constitutive g protein-coupled receptor activity. Annu Rev Pharmacol Toxicol 47:53-87.

Szkudlinski MW, Fremont V, Ronin C, Weintraub BD. 2002. Thyroid-stimulating hormone and thyroid-stimulating hormone receptor structure-function relationships. Physiol Rev 82:473-502.

Tao YX. 2006. Inactivating mutations of G protein-coupled receptors and diseases: structure-function insights and therapeutic implications. Pharmacol Ther 111:949-973.

Tao YX. 2008. Constitutive activation of G protein-coupled receptors and diseases: insights into mechanisms of activation and therapeutics. Pharmacol Ther 120:129-148.

Trulzsch B, Krohn K, Wonerow P, Chey S, Holzapfel HP, Ackermann F, Fuhrer D, Paschke R. 2001. Detection of thyroidstimulating hormone receptor and Gsalpha mutations: in 75 toxic thyroid nodules by denaturing gradient gel electrophoresis. J Mol Med 78:684-691.

Van Durme J, Horn F, Costagliola S, Vriend G, Vassart G. 2006. GRIS: glycoprotein-hormone receptor information system. Mol Endocrinol 20:2247-2255. 